\title{
Phraseologisches Optimum für Deutsch als Fremdsprache. Ein Vorschlag auf der Basis von Frequenz- und Geläufigkeitsuntersuchungen
}

\author{
Erla Hallsteinsdóttir (Odense)/Monika Šajánková (Bratislava)/Uwe Quasthoff (Leipzig)
}

\begin{abstract}
In this paper we introduce results from two studies on frequency and knowledge of German idioms. The aim of the first study was to determine the frequency of idioms in a written language corpus, and in the second study the native speakers knowledge of idioms was exploited. In both studies selected idioms from dictionaries of idioms and dictionaries for German as a foreign language were used, and here we present the result of the comparison of the data from both studies concerning 1112 German idioms. One part of the result is a list with 143 highly frequent and well known idioms, that we propose to be a part of a "phraseological optimum" for German as a foreign language.
\end{abstract}

\section{$1 \quad$ Einführung}

Die Frage nach dem phraseologischen "was" und "wie" für Deutsch als Fremdsprache beschäftigt die Phraseologieforschung schon seit einigen Jahren. Häufig wird in diesem Zusammenhang auf die Notwendigkeit der Erstellung phraseologischer und parömiologischer Minima bzw. Optima hingewiesen, in denen die Phraseologismen und Parömien aufgelistet und beschrieben werden, die ein Fremdsprachenlerner beherrschen sollte (cf. Hessky 1992). Die Frage nach phraseologischen Optima ist nicht nur für die Fremdsprachendidaktik sondern in hohem Maße auch für die Lernerlexikographie von Relevanz. Das "Phraseologie- und Idiomatizitätsverständnis" (Wotjak 2001: 264) der meisten Autoren deutscher Lernerwörterbücher ist bisher ein wohl gehütetes Geheimnis, zumindest was die Kriterien für die Auswahl von Phraseologismen für die Wörterbücher betrifft (cf. Wotjak 2001: 264). Da die verwendeten Wörterbücher jedoch für Deutsch als Fremdsprache verfasst wurden, liegt es nahe, die darin enthaltenen Phraseologismen in Bezug auf ihre Relevanz für ein phraseologisches Optimum für Deutsch als Fremdsprache zu untersuchen.

In diesem Beitrag werden die Ergebnisse aus zwei unabhängig von einander durchgeführten Untersuchungen verglichen und zueinander in Relation gesetzt. Es handelt sich um eine Untersuchung zur Frequenz deutscher Phraseologismen im Korpus Deutscher Wortschatz und eine Untersuchung zur Geläufigkeit deutscher Phraseologismen bei Muttersprachlern des Deutschen. Weiterhin wird auf den Ergebnissen des Vergleichs aufbauend eine Aufteilung der 
Phraseologismen für ein phraseologisches Optimum für Deutsch als Fremdsprache durchgeführt.

Im Folgenden wird für das untersuchte sprachliche Material der Terminus Phraseologismus verwendet. Es handelt sich hauptsächlich um die lexikalisierten Mehrwortverbindungen, die in der Forschung als "Phraseolexeme", "Wortgruppenidiome" oder "Phraseologismen" im engeren Sinne bezeichnet werden. Eine genauere Klassifikation ist aufgrund der uneinheitlichen Klassifikationen in den verwendeten Wörterbüchern nicht sinnvoll und für die hier verfolgten Ziele auch nicht notwendig.

\section{Frequenz und Geläufigkeit von Phraseologismen}

Methodische Überlegungen und Untersuchungen zur Geläufigkeit und zur Frequenz von Phraseologismen sind inzwischen ein wichtiger Bestandteil der Phraseologieforschung. Für die deutsche Phraseologie besteht in diesem Bereich jedoch nach wie vor ein Forschungsdesiderat. Die vorhandenen Untersuchungen in anderen Sprachen basieren in der Regel auf mindestens einem der folgenden Verfahren:

(a) Informantenbefragungen sind ein wichtiges Werkzeug zur Bestimmung der Geläufigkeit und der Bedeutung einzelner Phraseologismen im Bewusstsein der Sprecher. Eine Überprüfung des gesamten Phraseologiebestandes einer Sprache auf diese Art und Weise wäre allerdings im Hinblick auf die Kosten und den Arbeitsaufwand schwer zu bewältigen (cf. Čermák 2003).

(b) Korpusanalysen: Ergebnisse zur Frequenz von Phraseologismen als Angaben zur relativen Frequenz einzelner Phraseologismen auf eine Million Wörter (PMW-Angaben) liegen in einigen Sprachen wie Tschechisch (cf. Čermák 2003), Französisch, Niederländisch (cf. Colson 2003) und Englisch (cf. Cowie 2003) bereits vor. Diese Ergebnisse wurden in der Regel dadurch gewonnen, dass spezielle Wortkombinationen ausgewählt und auf ihr Vorkommen im Korpus überprüft wurden.

(c) Die statistische Kookkurenzanalyse ist ein Verfahren, mit dem vom Korpus ausgehend d. h. ohne Vorgabe von Wortkombinationen - die Frequenz von idiomatischen Wortverbindungen untersucht wird. Am Institut für Deutsche Sprache in Mannheim wird an einer statistischen Kookkurenzanalyse für die deutsche Sprache gearbeitet (cf. Steyer 2003).

(d) Die eigene Sprachkompetenz bzw. die Intuition der Linguisten ist ein umstrittenes Kriterium in der Frequenzforschung. Sie ist ohne Frage sehr subjektiv und individuell ausgeprägt - beim Linguisten zusätzlich vom Fachwissen und theoretischen Annahmen belastet. Die Sprachkompetenz ist jedoch nicht aus der Frequenzforschung zu verbannen, denn sowohl für Informantenbefragungen als auch für Korpusanalysen muss geeignetes Ausgangsmaterial gefunden und vorbereitet werden und dazu braucht der Linguist seine Intuition. Außerdem müssen die Ergebnisse einer Korpusanalyse auf der Basis der individuellen Sprachkompetenz ausgewertet und interpretiert werden, denn bei vielen Phraseologismen kann ein anderer Phraseologismus oder eine frei konstruierte 
für Deutsch als Fremdsprache.

Wortverbindung in derselben Kombination vorkommen, so dass nur eine manuelle Analyse die phraseologische und die freie Wortverbindung unterscheiden kann.

\subsection{Die Frequenzuntersuchung}

Die Frequenzuntersuchung ${ }^{1}$ wurde ursprünglich als Grundlage für die Auswahl von deutschen Phraseologismen für eine zweisprachige Phraseologiedatenbank durchgeführt (cf. Hallsteinsdóttir 2005 und im Druck a, b). Die Phraseologismen für die Untersuchung wurden den beiden zu diesem Zeitpunkt vorhandenen Wörterbüchern für Deutsch als Fremdsprache entnommen. Die im Wörterbuch Deutsch als Fremdsprache von de Gruyter (GDaF) (Kempcke 2000) mit einem Stern (*) markierten Phraseologismen bildeten das Ausgangsmaterial. Zwar werden im GDaF keine genauen Kriterien für die Auswahl von Phraseologismen angegeben. "Der Weg der Materialselektion bleibt also opak, ..." (Wotjak 2001: 269). Die Auswahl wird trotzdem - allerdings auch ohne genauere Angabe der Kriterien dafür - als gelungen und treffend angesehen:

Es wird also deutlich, dass das GDaF dem Lernenden ein sehr reichhaltiges, dabei aber durchaus geläufiges und nicht antiquiertes phraseologisches Material zur Verfügung stellt. (Wotjak 2001: 270)

Somit ist davon auszugehen, dass sich die Phraseologismen aus diesem Wörterbuch gut für eine erste Untersuchung zur Frequenz deutscher Phraseologismen eignen. Die Phraseologismen wurden ergänzt mit den mit ID markierten Phraseologismen aus dem Langenscheidt Wörterbuch für Deutsch als Fremdsprache (Götz et al. 1997), den Phraseologismen aus dem Wörter- und Übungsbuch von Hessky/Ettinger (1997) und aus dem phraseologischen Wörterbuch von Langenscheidt (Griesbach/Schulz 2000) sowie durch die Liste intersubjektiv geläufiger deutscher Idiome von Dobrovol'skij (1997b: 265-288). Mit dieser Auswahl an deutschen Phraseologismen sollte ein Großteil der häufigsten und geläufigsten Phraseologismen der deutschen Sprache erfasst sein. Insgesamt ergaben die Wörterbücher etwas über 6000 unterschiedliche Phraseologismen. Zu gut 5000 Phraseologismen konnten weitgehend eindeutige Suchformen manuell konstruiert werden, bei den übrigen 1000 handelt es sich hauptsächlich um Phraseologismen mit einer zu variablen Form, wie. z. B. einen Bart haben oder etwas für sich behalten .

Die Phraseologismen wurden in April 2002 im Korpus des Wortschatzlexikons Deutscher Wortschatz (www.wortschatz.uni-leipzig.de) auf ihr Vorkommen überprüft. Die technische Seite der Frequenzuntersuchung und die Zusammenstellung der Daten für weitere Analysen wurden von Mitarbeitern des Instituts für Informatik an der Universität Leipzig betreut. Das dem Wortschatzlexikon zugrunde liegende Korpus hatte zu diesem Zeitpunkt rund 21 Millionen Sätze mit 230 Millionen laufenden Wörtern. Die Ergebnisse dieser Untersuchung wurden in Mai/Juni 2005 durch weitere Untersuchungen in Deutscher Wortschatz ergänzt. Dabei wurden Phraseologismen aus der Geläufigkeitsuntersuchung ohne Frequenzangabe und

\footnotetext{
${ }^{1}$ Die Frequenzuntersuchung wurde finanziert von Rannís - The Icelandic Centre for Research (www.rannis.is). An dieser Stelle möchten wir uns für die finanzielle Unterstützung danken.
} 
Phraseologismen mit der Frequenzangabe null überprüft. Das Wortschatzlexikon hat unterdessen knapp doppelt so viel Text im Vergleich zu 2002, nämlich 35 Millionen Sätze mit 500 Millionen laufenden Wörtern. Die neuen Frequenzresultate wurden an die Ergebnisse aus der ersten Untersuchung angepasst, d. h. die absolute Anzahl an Belegen wurde halbiert (Stichproben der vorhandenen Ergebnisse aus der ersten Untersuchung haben dieses Verfahren bestätigt). Für die Phraseologismen aus der Geläufigkeitsuntersuchung (siehe unten), bei denen keine eindeutige Suchform möglich war, wurden Kollokationsangaben in Deutscher Wortschatz ausgewertet.

Frequenzuntersuchungen, wie sie hier vorgenommen wurden, ergeben nur eine erste Grundlage für die Auswahl von Phraseologismen für didaktische oder lexikographische Zwecke in Deutsch als Fremdsprache, sie sollten jedoch nicht das einzige Kriterium sein. Die Gründe dafür sind vor allem die folgenden:

- Das phraseologische Ausgangskorpus berücksichtigt - bedingt durch die Auswahl auf der Basis von Wörterbüchern für Deutsch als Fremdsprache - nur einen kleinen Teil der Phraseologie der deutschen Sprache.

- Die Frequenzangaben sind an die verwendeten Suchformen gebunden. Die Korpusbelege wurden bisher nur zum Teil ausgewertet. Die Form allein entscheidet also über die Frequenz. Dies bedeutet, dass in den Belegen nicht zwischen einer freien Lesart (i. e. einer freien Wortverbindung) und einem Phraseologismus unterschieden wird. Eine solche Unterscheidung muss durch eine manuelle Auswertung erfolgen. Die bisherigen Analysen lassen allerdings die Schlussfolgerung zu, dass in der Regel weniger als 10\% der Belege eine nicht-phraseologische Realisierung haben, ja dass sogar bei den meisten Phraseologismen eine nicht-phraseologische Bedeutung gar nicht vorkommt. Dies gilt auch für Phraseologismen wie grünes Licht geben, jm unter die Arme greifen, etw. auf Eis legen, gegen den Strom schwimmen (cf. Hallsteinsdóttir im Druck c).

- Bei der Analyse von Kollokationsangaben in Deutscher Wortschatz bei den Phraseologismen, zu denen keine eindeutige Suchform konstruiert werden konnte, ist auch noch keine manuelle Auswertung erfolgt.

- Die Suchformen wurden manuell konstruiert, d. h. sie basieren auf der Intuition eines Sprechers, und es könnten daher Suchformen fehlen bzw. Suchformen verwendet worden sein, die nicht nur den Ausgangsphraseologismus, sondern auch andere Phraseologismen oder freie Wortverbindungen erfassen. Außerdem können nicht für alle Phraseologismen brauchbare Suchformen konstruiert werden.

- Diese Untersuchung bezieht sich auf die geschriebene Sprache in dem verwendeten Leipziger Korpus und berücksichtigt nicht die gesprochene Sprache. Es können mit diesen Ergebnissen daher keine Angaben über den mündlichen Gebrauch von Phraseologismen gemacht werden.

Um diese Nachteile zumindest teilweise auszugleichen, müssen bei der Auswahl und Bearbeitung der Phraseologismen zusätzlich andere Verfahren eingesetzt werden. Ein solches Verfahren ist die empirische Befragung muttersprachlicher Sprecher nach der Bekanntheit 

für Deutsch als Fremdsprache.

von Phraseologismen. Eine solche Befragung mit 1112 deutschen Phraseologismen und 101 Teilnehmern wird im nächsten Kapitel vorgestellt.

\subsection{Die Geläufigkeitsuntersuchung}

Die Geläufigkeitsuntersuchung wurde im Rahmen eines Promotionsprojektes an der Universität Bratislava durchgeführt (cf. ausführlich in Šajánková 2005a). In einer Fragebogenuntersuchung wurden Probanden mit Deutsch als Muttersprache gebeten, den Bekanntheitsgrad deutscher Phraseologismen anzugeben. Das Ziel der Untersuchung war die Dokumentation der Geläufigkeit von Phraseologismen, die von deutschen Muttersprachlern, insbesondere in der Alltagskommunikation, aktiv verwendet werden. Die Ergebnisse sollen eine Grundlage für die Entwicklung der phraseologischen Kompetenz in der Fremdsprache Deutsch bilden.

Das Phraseologiekorpus der Fragebogenuntersuchung entstand durch den Vergleich von fünf phraseologischen Übungs- und Lernwörterbüchern (siehe Abbildung 1) und es wurde durch eigene Hörbelege ergänzt. Da es aus Ressourcengründen nicht möglich war, den Probanden alle Phraseologismen vorzulegen, wurden die Übungs- und Lernwörterbücher verglichen, und die am häufigsten vorkommenden Phraseologismen in den Fragebogen aufgenommen. Die Häufigkeit des Vorkommens in den Lehrwerken als Kriterium für die Auswahl für den Fragebogen $\mathrm{zu}$ verwenden, erwies sich jedoch als problematisch. Nur wenige Phraseologismen kamen in allen Lehrwerken vor und viele der verzeichneten Hörbelege wurden nur ein- oder zweimal aufgeführt. Nachdem kein zusätzliches Kriterium gefunden werden konnte, um sicherzustellen, dass nur unbekannte Phraseologismen entfernt würden, wurde das zweifache Vorkommen als Aufnahmekriterium festgelegt.

\begin{tabular}{|l|l|l|}
\hline Wörterbuch & $\begin{array}{l}\text { Insgesamt } \\
\text { im WB }\end{array}$ & $\begin{array}{l}\text { im } \\
\text { Fragebogen }\end{array}$ \\
\hline $\begin{array}{l}\text { Hessky, Regina/Ettinger, Stefan (1997): Deutsche Redewendungen. } \\
\text { Ein Wörter- und Übungsbuch für Fortgeschrittene. }\end{array}$ & ca. 1400 & 822 \\
\hline $\begin{array}{l}\text { Wotjak, Barbara/Richter, Manfred (1993): Sage und schreibe. } \\
\text { Deutsche Phraseologismen in Theorie und Praxis. }\end{array}$ & ca. 850 & 517 \\
\hline $\begin{array}{l}\text { Griesbach, Heinz/Uhlich, Gudrun (1993): Mit anderen Worten. } \\
\text { Deutsche Idiomatik. Redensarten und Redeweisen. }\end{array}$ & ca. 400 & 727 \\
\hline $\begin{array}{l}\text { Herzog, Annelies (1993): Idiomatische Redewendungen von A-Z. } \\
\text { Ein Übungsbuch für Anfünger und Fortgeschrittene. }\end{array}$ & ca. 600 & 282 \\
\hline $\begin{array}{l}\text { Dobrovo'skij, Dimitrij (1997b): Idiome der Lebendigen Sprache. } \\
\text { Deutsch - Russisches Wörterbuch. }\end{array}$ & ca. 1000 & 604 \\
\hline $\begin{array}{l}\text { Buscha, Joachim (1979): Deutsches Übungsbuch. } 100 \\
\text { Phraseologismen in Bildern. }\end{array}$ & 100 & 96 \\
\hline Hörbelege & 163 & 163 \\
\hline
\end{tabular}

Abbildung 1: In der Geläufigkeitsuntersuchung verwendete Wörterbücher 
Der Fragebogen wurde in Anlehnung an vorhandene Untersuchungen zur Bekanntheit von Sprichwörtern (cf. Chlosta et al. 1994M; Ďurčo 2001) und Phraseologismen (cf. Durčo 1994; Hallsteinsdóttir 2001) entwickelt. In dem Fragebogen wurden die Phraseologismen mit folgenden Antwortmöglichkeiten versehen, die von den Probanden angekreuzt werden sollten. Nur bei Antwortmöglichkeit 4 wurde um eigene, frei formulierte Angaben zu bekannten Varianten gebeten.

1. Ich kenne diese Redensart nicht.

2. Ich kenne diese Redensart (habe sie schon gehört/gelesen), aber ich verwende sie selbst in meiner eigener Sprache nicht.

3. Ich kenne diese Redensart und verwende sie selbst, (oder ich könnte mir durchaus vorstellen, dass ich sie in bestimmten Situationen verwenden würde).

4. Ich kenne eine andere Variante und a) verwende sie; b) verwende sie nicht

Abbildung 2: Antwortmöglichkeiten im Fragebogen zur Geläufigkeit deutscher Phraseologismen

Hierbei ist noch zu bemerken, dass es einfacher zu entscheiden ist, ob ein Phraseologismus bekannt ist, d. h. dass ihm der Sprecher schon mal im Sprachverkehr begegnet ist (gehört oder gelesen hat), als die Frage zu beantworten, ob er auch in der eigenen Rede gebräuchlich ist. Dazu ist ein gewisser Grad an Sprachreflexion erforderlich, denn die einzelnen Einträge werden rein subjektiv und metakommunikativ ohne sprachlichen Kontext eingeschätzt. Mit dieser Vorgehensweise ist auch die Frage verbunden, inwieweit ein Sprecher überhaupt fähig ist, den eigenen Sprachgebrauch zu reflektieren. Zusätzlich stellt sich die Frage, ob seine Einschätzung bei derart umfangreichen Fragebogen nicht rein mechanisch durchgeführt wird bzw. es besteht die Gefahr, dass das Interesse und die metasprachliche Reflexion im Laufe der Bearbeitung stark nachlassen. Solche individuell bedingten Faktoren stellen allerdings bei fast jeder empirischen Befragung ein gewisses Risiko dar.

Der Fragebogen wurde per E-Mail an verschiedene Universitäten in Deutschland geschickt, mit der Bitte ihn an möglichst viele potenzielle Probanden weiter zu verteilen. Insgesamt haben 101 Probanden den Fragebogen ausgefüllt zurückgeschickt. Bei der Auswertung wurde der Durchschnittswert der Geläufigkeit bei jedem Phraseologismus für jede Antwortmöglichkeit ermittelt (0-101). Dieser Wert geht in die vergleichende Untersuchung ein. Die Antwortmöglichkeit "andere Variante bekannt" wird bei dem Vergleich der Untersuchungen nicht berücksichtigt, denn bei dieser Antwort werden keine Angaben zur Geläufigkeit der bekannten Variante gemacht.

Da das Alter für die Ausprägung der phraseologischen Kompetenz eine Rolle spielen kann, soll hier auf die Altersverteilung der Probanden hingewiesen werden: 64 Probanden sind jünger als 30 Jahre und 37 sind älter als 30 Jahre. An der Umfrage haben also verhältnismäßig viele jüngere Probanden (Studenten oder junge Universitätsabsolventen) teilgenommen. 
für Deutsch als Fremdsprache.

Der Fragebogen beinhaltet insgesamt 1179 Phraseologismen. Zu 1112 davon liegen Daten aus der Frequenzuntersuchung vor. Die Geläufigkeit dieser Phraseologismen verteilt sich wie folgt:

624 Phraseologismen werden aktiv verwendet von 76-101 Probanden

335 Phraseologismen werden aktiv verwendet von 51-75 Probanden

113 Phraseologismen werden aktiv verwendet von 26-50 Probanden

40 Phraseologismen werden aktiv verwendet von 0-25 Probanden

Diese Phraseologismen bilden das Phraseologiekorpus für die vergleichende Untersuchung von Geläufigkeit und Frequenz.

\section{Phraseologische Optima für den Fremdsprachenunterricht}

Schon seit langem wird auf die Notwendigkeit der Bestimmung und Beschreibung der Phraseologismen hingewiesen, die Fremdsprachenlerner beherrschen sollen (cf. z. B. Eismann 1979, Hessky 1992 und 1997b). Überlegungen zur Festlegung eines phraseologischen Minimums bzw. Optimums (cf. Hessky 1997a: 139) kommen in der Regel aus der Fremdsprachendidaktik (cf. Darstellung in Hessky 1992: 159 ff.). So fordert Hessky eine Liste von Phraseologismen für Deutsch als Fremdsprache, die jeweils "in Abhängigkeit von der Muttersprache der Lernenden, auf kontrastiver Basis und unter Zielsetzung, Lernstufe etc." (Hessky 1992: 167) für den Unterricht aufgearbeitet werden müssen.

Ein weiterer Bereich, in dem ein phraseologisches Optimum bisher selten thematisiert wurde, für den dessen Bestimmung aber höchst relevant ist, ist die Fremdsprachenlexikographie. In phraseologischen Lern- und Wörterbüchern wird die Auswahl der Phraseologismen zwar immer gut begründet,

[a]ngeblich werden die Beispiele nach Frequenz, Disponibilität, Wichtigkeit, Kontrastivität, Benutzer- bzw. Adressatenbezug usw. ausgewählt, in Wirklichkeit ist ihre Auswahl jedoch recht willkürlich. (Ettinger 1998: 203)

Die Vorarbeiten für unsere Untersuchungen haben dies bestätigt, indem es sich herausgestellt hat, dass nur wenige Phraseologismen in mehreren Wörterbüchern vorkommen. Ein definiertes phraseologisches Optimum mit genauen Beschreibungen der Phraseologismen und Angaben zur Geläufigkeit und Frequenz würde eine einheitliche Grundlage für die Lernerlexikographie ergeben (cf. Dobrovol'skij 1997b: 50 und Hallsteinsdóttir 2006). Für beide diese Bereiche gilt außerdem, dass ein definiertes Optimum dazu führen würde, dass seltene und unbekannte Phraseologismen aus den Lehrwerken bzw. Wörterbüchern verschwinden. Es würde

... wohl ermöglichen, daß man abkommt von dem reflexartig praktizierten Vorgehen, daß "bildhafte" Redensarten - mitunter völlig unabhängig von ihrem kommunikativen Stellenwert in Lehrmaterialien mehr oder weniger gleichmäßig verteilt wie Rosinen präsentiert, um den "Kuchen Fremdsprache" den Lernenden dadurch schmackhafter zu machen. (Hessky 1992: 161) 
Es handelt sich dabei um Phraseologismen wie Eulen nach Athen tragen ${ }^{2}$, Stein und Bein frieren, jm um den Bart gehen, jm goldene Berge versprechen, saufen wie ein Bürstenbinder, die sowohl den hier befragten Muttersprachlern wenig geläufig sind, als auch nur selten im Korpus vorkommen (cf. dazu auch Hallsteinsdóttir 2001).

Bei Ďurčo (2001: 100) werden fünf verschiedene Herangehensweisen bei der Erstellung von phraseologischen Minima dargestellt. Diese haben bei Untersuchungen zu Parömien zwar teilweise zu sehr unterschiedlichen Ergebnissen geführt, wir sind aber der Meinung, dass sie in der richtigen Kombination eine verlässliche Grundlage für die Bestimmung phraseologischer Optima liefern können, wie wir im nächsten Kapitel zeigen. Die Herangehensweisen sind:

- Analysen von Publikationen und Wörterbüchern. Da Publikationen (Forschungsliteratur, Lehrwerke und/oder Wörterbücher) immer subjektive (und meist nicht genauer erläuterten) Entscheidungen der jeweiligen Autoren zu Grunde liegen, sind solche Analysen nur sehr eingeschränkt für die Bestimmung phraseologischer Optima geeignet, sie können höchstens das Material für weitere Untersuchungen liefern (siehe oben).

- Korpusanalysen. Eine Korpusanalyse kann wichtige Informationen zur Frequenz von Phraseologismen liefern. Sie kann jedoch nicht alleine eine Grundlage für ein phraseologisches Optimum bilden, denn sie sagt nur etwas über das Vorkommen von Phraseologismen (genauer gesagt: das Vorkommen von konstruierten Suchformen) in den Texten der verwendeten Korpora aus.

- Mit psycholinguistischen Experimenten und soziolinguistisch-empirischen Untersuchungen (Evokationstests: Listen mit konkreten Phraseologismen, zu denen Angaben gemacht werden) können Daten zur Geläufigkeit von Phraseologismen bei Sprechern gewonnen werden. Solche Daten stellen eine wichtige Ergänzung zu Resultaten aus Korpusanalysen dar.

\section{$4 \quad$ Ergebnisse der Untersuchungen}

Die Auswertung der Ergebnisse beider Untersuchungen bezieht sich nur auf die 1112 Phraseologismen, zu denen in den Untersuchungen Daten vorliegen; es handelt sich dabei überwiegend um Phraseologismen mit mindestens einem Substantiv als Komponente. Die Frequenz dieser Phraseologismengruppen wird in Abbildung 3 in Relation zur Geläufigkeit dargestellt:

\footnotetext{
${ }^{2}$ Ein Paradebeispiel, das sowohl im Unterricht Deutsch als Fremdsprache als auch in der (kontrastiven) Phraseologieforschung immer wieder auftaucht. Der Phraseologismus ist aber selten: die Suchform "Eulen nach Athen" kommt in Deutscher Wortschatz gerade 28 Mal vor und nur 33 der hier Befragten verwenden den Phraseologismus aktiv. In Hallsteinsdóttir (2001: 153) wurde der Phraseologismus sogar von keinem der befragten 41 deutschen Germanistikstudenten als "bekannt, aktiv verwendet" markiert, knapp die Hälfte hat ihn dagegen als "unbekannt" eingestuft.
} 


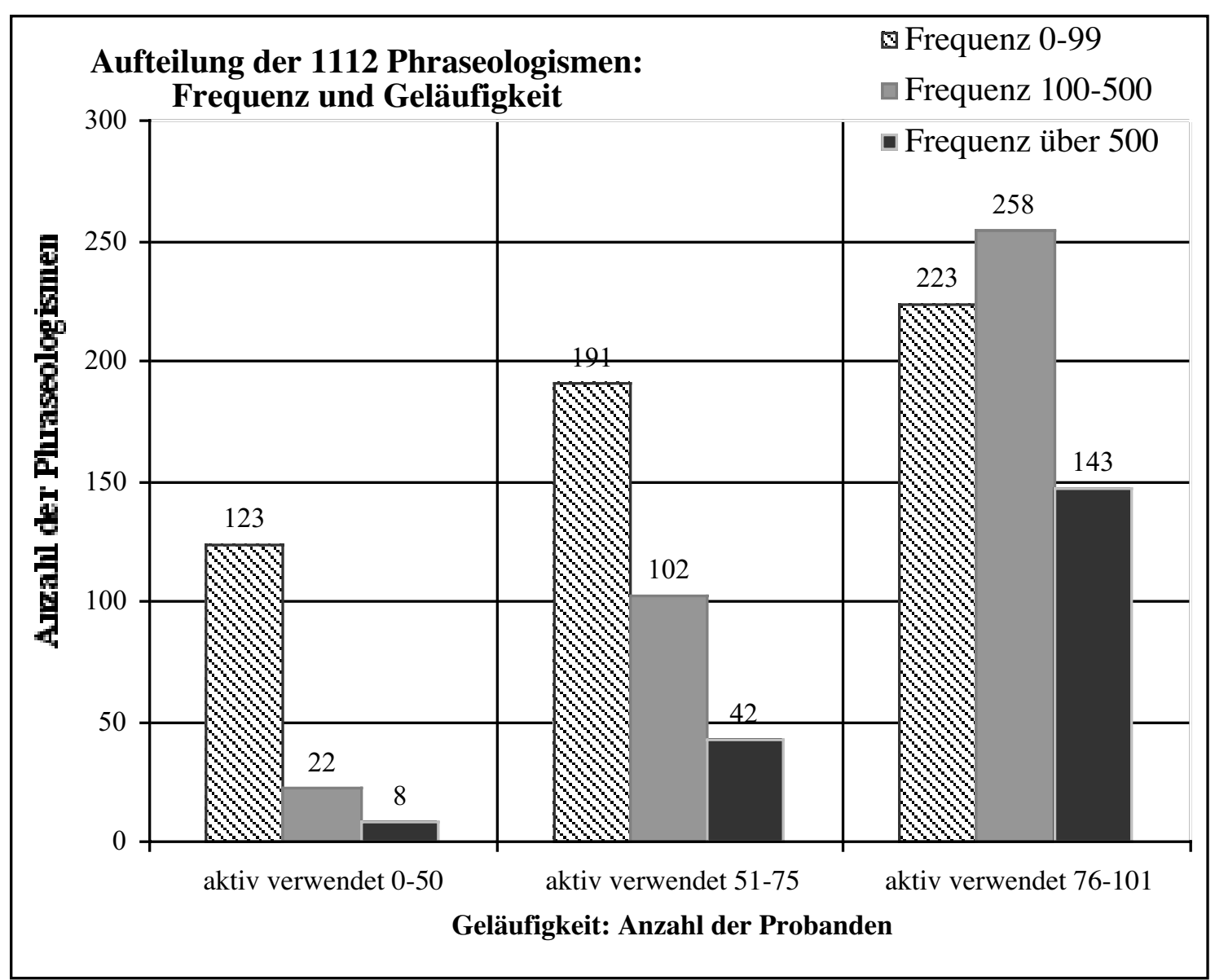

Abbildung 3: Antwortmöglichkeiten im Fragebogen zur Geläufigkeit deutscher Phraseologismen

Es gibt eine Korrelation zwischen Geläufigkeit und Frequenz, in dem Sinne dass der Großteil der Phraseologismen, die im Korpus eine hohe oder mittlere Frequenz haben (über 100 Belegstellen), auch von vielen Probanden aktiv verwendet wird. Außerdem weisen nur 30 der 153 Phraseologismen, die von weniger als der Hälfte der Probanden aktiv verwendet werden, eine höhere Frequenz als 100 Belegstellen auf. Die relativ hohe Anzahl aktiv verwendeter Phraseologismen (ca. 1/3 der von 76-101 Probanden aktiv verwendeten Phraseologismen), die wenige Belegstellen im Korpus haben, kann vermutlich teilweise dadurch erklärt werden, dass es sich um Phraseologismen handelt, die überwiegend im mündlichen Sprachgebrauch verwendet werden. Das Leipziger Korpus besteht aus geschriebenen Texten, in denen solche Phraseologismen naturgemäß nicht häufig vorkommen.

Die hier vorhandene Korrelation zwischen Frequenz und Geläufigkeit (viele Belegstellen hohe Geläufigkeit) bei Phraseologismen entspricht nicht den Ergebnissen aus Durčos Untersuchungen zur Frequenz von Sprichwörtern im Korpus und zur Geläufigkeit bei Muttersprachlern. In seinen Untersuchungen

... hat sich das erwartete Ergebnis darin bestätigt, dass der Bekanntheitsgrad, die lexikographische Erfassung und die textuelle Vorkommenshäufigkeit keine korrelierenden Werte sind. (Ďurčo 2001: 102) 
Eine Erwartung, dass es eine Korrelation gibt zwischen dem Vorkommen eines Phraseologismus im Wörterbuch, das in der Regel auf der individuellen Intuition der Verfasser basiert, und den anderen beiden Aspekten, der Frequenz im Korpus und der Geläufigkeit bei Sprechern, hat es aus unserer Sicht ohnehin nie gegeben. Es hat sich gleich bei der Auswertung der Wörterbücher gezeigt, dass es nur eine geringe Übereinstimmung in der Auswahl der Phraseologismen in den Wörterbüchern gibt.

Bei der Festlegung der Kriterien für die Gruppeneinteilung stößt man auf die Problematik, dass die Bestimmung, wann ein Phraseologismus als "frequent" oder "geläufig" anzusehen ist, einen gewissen Grad an Beliebigkeit aufweist. Die an sich "willkürliche" Einteilung in drei Gruppen (weniger als 100, 100-500 und über 500 Belegstellen) wird folgendermaßen begründet. Das phraseologische Ausgangskorpus für die Frequenzuntersuchung hatte ursprünglich 6000 Phraseologismen aus Wörterbüchern und Lehrwerken für Deutsch als Fremdsprache. Wenn man davon ausgeht, dass diese 6000 Phraseologismen ungefähr dieselbe Einteilung in Gruppen erreichen würden, wie die 1112 hier ausgewerteten Phraseologismen (13\% haben eine hohe Geläufigkeit und hohe Frequenz und gehören zum Kernbereich eines phraseologischen Optimums), dann bekäme man in etwa 800-1000 Phraseologismen, die den Kernbereich des phraseologischen Optimums für Deutsch als Fremdsprache bilden.

Die Anzahl geläufiger deutscher Idiome bewegt sich um 1000. Dies zeugt davon, daß in der Idiomatik ein intersubjektiv gültiger Kernbereich existiert, der auf empirischen Wegen ermittelt und mit verschiedenen Methoden verifiziert werden kann. (Dobrovol'skij 1997b: 50)

In Anlehnung an die Tabelle für die Auswertung des Bekanntheitsgrades von Sprichwörtern bei Ďurčo (2001: 103) werden die Phraseologismen im Folgenden nach ihrer Frequenz im Korpus und Geläufigkeit bei Muttersprachlern in Gruppen zusammengefasst.

\begin{tabular}{|c|c|c|}
\hline Vorkommen & Text & Usus \\
\hline Hoch & A & B \\
\hline Niedrig & C & D \\
\hline
\end{tabular}

Abbildung 4: Ďurčos Tabelle für die Auswertung des Bekanntheitsgrades von Sprichwörtern

Ďurčos Gruppierung enthält jedoch nur eine Gruppe (AB), die für Fremdsprachenlerner relevant ist. Die Typen AD und CB bezeichnet er als philologische Stufe, "die praktisch nur für die Studenten des entsprechenden philologischen Studiums in Frage käme." (Ďurčo 2001: 104). Hier wird daher eine etwas differenziertere Einteilung in neun Gruppen gewählt, um die Möglichkeit zwischen Phraseologismen für die aktive und Phraseologismen für die passive phraseologische Kompetenz zu wählen, die für den mündlichen und/oder für den schriftlichen Sprachgebrauch wichtig sind.

\begin{tabular}{|l|l|l|}
\hline $\begin{array}{l}\text { Frequenz und } \\
\text { Geläufigkeit }\end{array}$ & Frequenz im Korpus & $\begin{array}{l}\text { Geläufigkeit bei } \\
\text { Muttersprachlern }\end{array}$ \\
\hline Hoch & A über 500 Belegstellen & B 76-101 aktiv verwendet \\
\hline Mittel & C 101-500 Belegstellen & D 51-75 aktiv verwendet \\
\hline Niedrig & E 0-100 Belegstellen & F 0-50 aktiv verwendet \\
\hline
\end{tabular}



für Deutsch als Fremdsprache.

Abbildung 5: Erweiterte Tabelle für die Auswertung der Geläufigkeit und der Frequenz von Phraseologismen

Diese Einteilung wird im Hinblick auf die Relevanz der Gruppen für ein phraseologisches Optimum für Deutsch als Fremdsprache wie folgt begründet:

1. Gruppe AB - Zum Kernbereich eines phraseologischen Optimums für Deutsch als Fremdsprache gehören 143 Phraseologismen mit hoher Frequenz (über 500 Belege) und hoher Geläufigkeit (76-101). Diese Phraseologismen sollten im Grundwortschatz enthalten sein.

2. Gruppe CB - Zum Kernbereich des phraseologischen Optimums gehören auch die 258 Phraseologismen mit mittlerer Frequenz (100-500 Belege) und hoher Geläufigkeit (76-101). Diese sollten im Bereich der Sprachproduktion für gesprochene Texte und im Bereich der Sprachrezeption für geschriebene Texte vermittelt werden. Bsp.: nicht auf den Mund gefallen sein, ein Tropfen auf den heißen Stein sein.

3. Gruppe EB - Es ist anzunehmen, dass die 223 Phraseologismen, die eine niedrige Frequenz (weniger als 100 Belege) im Textkorpus und eine hohe Geläufigkeit (76-101) bei den Muttersprachlern aufweisen, zum großen Teil zur gesprochenen Sprache gehören. Wenn dies zutrifft, dann gehören diese Phraseologismen ebenso zum Kernbereich eines phraseologischen Optimums, zum Bereich der Sprachrezeption für die gesprochene Sprache, bei fortgeschrittenen Sprachlernern zum Bereich der Sprachproduktion. Bsp.: (bei jm) ins Fettnäpfchen treten, wie ein Wasserfall reden.

4. Gruppe AD - Die 42 Phraseologismen mit hoher Frequenz (über 500 Belege) und mittlerer Geläufigkeit (50-75) gehören zur Peripherie des phraseologischen Optimums, im Bereich der Sprachrezeption für geschriebene Texte. Bsp.: die Weichen für jn/etw. stellen, bei jm (tief) in der Kreide stehen.

5. Gruppe CD - Die 102 Phraseologismen mit mittlerer Frequenz (100-500 Belege) und mittlerer Geläufigkeit (51-75) gehören nicht zum phraseologischen Optimum, sie sollten jedoch zur passiven phraseologischen Kompetenz bei fortgeschrittenen Sprachlernern gehören. Bsp.: etw. auf die hohe Kante legen, jm den Wind aus den Segeln nehmen.

6. Gruppe ED - Die 191 Phraseologismen mit niedriger Frequenz (weniger als 100 Belege) und mittlerer Geläufigkeit (51-75) gehören nicht zum phraseologischen Optimum. Bsp.: Gift und Galle spucken/speien, schwört Stein und Bein (auf etw.) schwören.

7. Gruppe AF - Diese Gruppe von 8 Phraseologismen mit hoher Frequenz (über 500 Belege) und niedriger Geläufigkeit (0-50) muss genauer untersucht werden. Wenn die Angaben richtig sind, dann gehören diese Phraseologismen zur Peripherie des phraseologischen Optimums, zur passiven phraseologischen Kompetenz. Bsp.: mit dem Rücken an die Wand kommen, sich $_{\mathrm{Akk} .}$ im Sattel halten. 
8. Gruppe CF - Die Gruppe von 22 Phraseologismen mit mittlerer Frequenz (100-500 Belege) und niedriger Geläufigkeit (0-50) gehört nicht zum phraseologischen Optimum. Bsp.: jm an die Kandare nehmen, jm blauen Dunst vormachen.

9. Gruppe EF - Die 123 Phraseologismen mit niedriger Frequenz (weniger als 100 Belege) und niedriger Geläufigkeit (0-50) gehören nicht zum phraseologischen Optimum. Bsp.: mit Kanonen nach/auf Spatzen schießen, weder Fisch noch Fleisch sein.

Mit dieser Einteilung schließen wir uns Ďurčos (2001: 104) Schlussfolgerungen an,

- dass ein phraseologisches Optimum als "ein Extrakt der Korrelation zwischen Bekanntheit und Häufigkeit" bestimmt werden kann, und

- dass ein phraseologisches Optimum "als graduelle Kategorie angesehen werden [kann], d. h. in Abhängigkeit von der Stufe und Etappe des Deutschlernens" in Relation zu den erwünschten sprachlichen Fertigkeiten bestimmt werden sollte (cf. dazu auch Hessky 1992 und 1997b).

Wir möchten auf der Grundlage der neun aufgelisteten Gruppen vorschlagen, die Phraseologismen aus Gruppe $A B$ als den offenen Kernbereich eines phraseologischen Optimums für Deutsch als Fremdsprache anzusehen. Die Liste im Anhang dieses Beitrags beinhaltet alle 143 Phraseologismen aus dieser Gruppe ${ }^{3}$. Es handelt sich um die Phraseologismen mit hoher Frequenz (über 500 Belege) und hoher Geläufigkeit (76-101), die auf jeden Fall zum Kernbereich eines phraseologischen Optimums für Deutsch als Fremdsprache gehören sollten. Da wir nur einen kleinen Teil der deutschen Phraseologie untersucht haben, gehen wir allerdings nicht davon aus, dass dies die einzigen Phraseologismen sind, die zu dieser Gruppe gehören sollten.

Es sind bei der Auswertung der Untersuchungsergebnisse noch keine lexikalischsemantischen Relationen oder andere Gruppierungen in der Phraseologie (cf. dazu z.B. Hallsteinsdóttir 2001 und Roos 2001: 80 ff.) wie z. B. die Relation zwischen sich ein Bild von jm/etw. machen, (über etw.) im Bilde sein und jn (über etw.) ins Bild setzen berücksichtigt worden, daher wird nur der in der Geläufigkeitsuntersuchung verwendete Phraseologismus aufgelistet. Die Liste ist nach der ersten substantivischen Komponente alphabetisch sortiert.

Um die in der Liste aufgeführten Phraseologismen in Wörterbücher aufzunehmen oder im Fremdsprachenunterricht einzusetzen, ist $u$. a. eine ausführliche lexikographische Beschreibung, die Durchführung intersprachlicher Vergleiche und eine didaktische Aufarbeitung notwendig (cf. Jesenšek im Druck a). Die Arbeit an der mehrsprachigen lexikographischen Erfassung (cf. Jesenšek im Druck b und www.ephras.com) an und der Didaktisierung (cf. Śajánková im Druck) dieser Phraseologismen hat bereits angefangen.

\footnotetext{
${ }^{3}$ Die Listen mit den Phraseologismen aus den anderen Gruppen können unter erlahall@yahoo.dk angefordert werden.
} 


\section{$5 \quad$ Zusammenfassung und Ausblick}

In diesem Beitrag haben wir einen ersten Vorschlag für den Kernbereich eines phraseologischen Optimums für Deutsch als Fremdsprache gemacht. Diese Phraseologismen sollen und können allerdings kein endgültig abgeschlossenes phraseologisches Optimum für Deutsch als Fremdsprache darstellen, denn es wurde zugegebenermaßen nur ein kleiner Ausschnitt der deutschen Phraseologie untersucht.

Das von uns vorgeschlagene Optimum ist als eine offene Liste von Phraseologismen anzusehen, die u.a. in Relation zum Lernziel und abhängig von der Muttersprache der jeweiligen DaF-Lerner modifiziert werden sollte. Die Phraseologismen müssen außerdem noch mit einer genauen Beschreibung versehen werden. Erst dann stellen sie eine Grundlage dar, die im Fremdsprachenunterricht oder für lexikographische und kontrastive Arbeiten zur Verfügung verwendet werden kann.

Wir sind uns der Grenzen der verwendeten Methodik und Datengrundlage (Anzahl der befragten Personen, Spezifika des verwendeten Korpus, Auswahl der Phraseologismen) durchaus bewusst. Die Ergebnisse halten wir trotzdem für aussagekräftig und die hier vorgeschlagenen Phraseologismen des Optimums für den Einsatz im DaF-Unterricht für ausreichend abgesichert. Wir betrachten diesen Beitrag als einen ersten Schritt auf dem Weg, konkretes phraseologisches Material für den Fremdsprachenunterricht aufzuarbeiten. Eine spezifischere didaktische Aufarbeitung muss in jedem Falle noch erfolgen. Ebenso betrachten wir unsere Arbeit als einen Beitrag zu einer grundlegenden theoretisch-methodischen Diskussion zur Methodik, Möglichkeiten und Zielen der Korpuslinguistik in Relation zur angewandten Linguistik.

\section{Literaturangaben}

Buscha, Joachim (1979): Deutsches Übungsbuch. 100 Phraseologismen in Bildern. Leipzig. Čermák, František (2003): "Paremiological Minimum of Czech: The Corpus Evidence". In: Burger, Harald/Häcki Buhofer, Annelies/Gréciano, Gertrud (eds.): Flut von Texten Vielfalt der Kulturen. Baltmannsweiler: 15-31. (= Phraseologie und Parömiologie 14).

Chlosta, Christoph/Grzybek, Peter/Ross, Undine (1994): "Wer kennt denn heute noch den Simrock? Ergebnisse einer empirischen Untersuchung zur Bekanntheit deutscher Sprichwörter in traditionellen Sammlungen." In: Chlosta, Christoph/Grzybek, Peter/Piirainen, Elisabeth (eds.): Sprachliche Bilder zwischen Theorie und Praxis. Bochum: 31-60. (= Studien zur Phraseologie und Parömiologie 2).

Colson, Jean-Pierre (2003): "Corpus Linguistics and Phraseological Statistics: a few Hypotheses and Examples." In: Burger, Harald/Häcki Buhofer, Annelies/Gréciano, Gertrud (eds.): Flut von Texten - Vielfalt der Kulturen. Baltmannsweiler: 47-59. (= Phraseologie und Parömiologie 14).

Cowie, Anthony P. (2003): "Exploring native-speaker knowledge of phraseology: informant testing or corpus research?" In: Burger, Harald/Häcki Buhofer, Annelies/Gréciano, Gertrud 
(eds.): Flut von Texten - Vielfalt der Kulturen. Baltmannsweiler: 73-81. (= Phraseologie und Parömiologie 14).

Dobrovol'skij, Dmitrij O. (1997a): Idiome der lebendigen Sprache. Deutsch-russisches Wörterbuch. Moskva.

Dobrovol'skij, Dmitrij O. (1997b): Idiome im mentalen Lexikon. Ziele und Methoden der kognitivbasierten Phraseologieforschung. Trier. (= Fokus 18).

Ďurčo, Peter (1994): Probleme der allgemeinen und kontrastiven Phraseologie: Am Beispiel Deutsch und Slowakisch. Heidelberg.

Ďurčo, Peter (2001): "Bekanntheit, Häufigkeit und lexikographische Erfassung von Sprichwörtern. Zu parömiologischen Minima für DaF". In: Häcki Buhofer, Annelies/Burger, Harald/Laurent, Gautier (eds.): Phraseologiae Amor. Aspekte europäischer Phraseologie. Baltmannsweiler, Schneider Verlag: 99-106. (= Phraseologie und Parömiologie 8).

Ďurčo, Peter (2003): "Empirische Daten zur Sprichwortvariabilität". In: Burger, Harald/Häcki Buhofer, Annelies/Gréciano, Gertrud (eds.): Flut von Texten - Vielfalt der Kulturen. Baltmannsweiler: 83-95. (= Phraseologie und Parömiologie 14).

Eismann, Wolfgang (1979): "Phraseologie und Russischunterricht in der Sowjetunion". Die Neueren Sprachen 78/6: 555-572.

Ettinger, Stefan (1998): "Einige Überlegungen zur Phraseodidaktik". In: Eismann, Wolfgang (ed.): EUROPHRAS 95. Europäische Phraseologie im Vergleich: Gemeinsames Erbe und kulturelle Vielfalt. Bochum: 201-217. (= Studien zur Phraseologie und Parömiologie 15).

Götz, Dieter/Haensch, Günther/Wellmann, Hans (eds.) $\left({ }^{5} 1997\right)$ : Langenscheidts Großwörterbuch Deutsch als Fremdspache. Berlin etc.

Griesbach, Heinz/Schulz, Dora (2000): 1000 deutsche Redensarten. Mit Erklärungen und Anwendungsbeispielen. Berlin et.

Griesbach, Heinz/Uhlich, Gudrun (1993): Mit anderen Worten. Deutsche Idiomatik. Redensarten und Redeweisen. München.

Hallsteinsdóttir, Erla (2001): Das Verstehen idiomatischer Phraseologismen in der Fremdsprache Deutsch. Hamburg (= PHILOLOGIA - Sprachwissenschaftliche Forschungsergebnisse 49). http://www.verlagkovac.de/0435_volltext.htm.

Hallsteinsdóttir, Erla (2003): "Das Verstehen idiomatischer Phraseologismen in der Fremdsprache Deutsch". In: Burger, Harald/Häcki Buhofer, Annelies/Gréciano, Gertrud (eds.): Flut von Texten - Vielfalt der Kulturen. Baltmannsweiler: 357-367. (= Phraseologie und Parömiologie 14).

Hallsteinsdóttir, Erla (2005): "Vom Wörterbuch zum Text zum Lexikon". In: Fix, Ulla et al. (eds.): Zwischen Lexikon und Text - lexikalische, stilistische und textlinguistische Aspekte. Leipzig: 325-337.

Hallsteinsdóttir, Erla (2006): "Phraseographie". HERMES Journal of Language and Communication Studies 36: 91-128.

Hallsteinsdóttir, Erla (im Druck: a): "A bilingual electronic dictionary of idioms". In: Gottlieb, Henrik/Mogensen, Jens Erik (eds.): Dictionaries, Lexicographical Options and User Needs. Amsterdam. (= Terminology and Lexicography Research and Practice). 

für Deutsch als Fremdsprache.

Hallsteinsdóttir, Erla (im Druck: b): "Konzeption und Erstellung einer computergestützten zweisprachigen Phraseologiesammlung Isländisch - Deutsch". In: Häcki Buhofer, Annelies/Burger, Harald (eds.): Phraseology in Motion. Proceedings of Europhras Basel 2004. Baltmannsweiler.

Hallsteinsdóttir, Erla (im Druck c): "Wörtliche, freie und phraseologische Bedeutung. Eine korpusbasierte Untersuchung des Vorkommens von freien und phraseologischen Lesarten bei deutschen Phraseologismen". In: Kržišnik, Erika (ed.): Phraseologie in der Sprachwissenschaft und anderen Disziplinen. Tagungsband EUROPHRAS Slovenija 2005.

Herzog, Annelies (u. Mitarbeit von Arthur Michel und Herbert Riedel) (1993): Idiomatische Redewendungen von A - Z. Ein Übungsbuch für Anfänger und Fortgeschrittene. Leipzig.

Hessky, Regina (1992): "Aspekte der Verwendung von Phraseologismen im Unterricht Deutsch als Fremdsprache". Fremdsprachen Lehren und Lernen 21: 159-168.

Hessky, Regina (1997a): "Feste Wendungen - ein heißes Eisen? Einige phraseodidaktische Überlegungen für den DaF-Unterricht". Deutsch als Fremdsprache 3/1997: 139-143.

Hessky, Regina (1997b): "Einige Fragen der Vermittlung von Phraseologie im Unterricht Deutsch als Fremdsprache". In: Wimmer, Rainer/Berens, Franz-Joseph (eds.): Wortbildung und Phraseologie. Tübingen: 255-261. (= Studien zur deutschen Sprache 9).

Hessky, Regina/Ettinger, Stefan (1997): Deutsche Redewendungen. Ein Wörter- und Übungsbuch für Fortgeschrittene. Tübingen.

Jesenšek, Vida (im Druck a): "Phraseologie in der Fremdsprache Deutsch". In: Begegnungssprache Deutsch, Band 1. Wien. Erscheint 2006.

Jesenšek, Vida (im Druck b): "EPHRAS. Mehrsprachige phraseologische Lernmaterialien auf CD-ROM". In: Kržišnik, Erika (ed.): Phraseologie in der Sprachwissenschaft und anderen Disziplinen. Tagungsband EUROPHRAS Slovenija 2005.

Kempcke, Günter (u. Mitarbeit von Barbara Seelig, Birgit Wolf, Elke Tellenbach et al.) (2000): Wörterbuch Deutsch als Fremdsprache. Berlin, New York.

Roos, Eckhard (2001): Idiom und Idiomatik: Ein sprachliches Phänomen im Lichte der kognitiven Linguistik und Gestalttheorie. Aachen. (= Sprache \& Kultur 39).

Šajánková, Monika (2004): "Phraseologismen im Fremdsprachenunterricht". In: Piirainen, Ilpo T./Meier, Jörg (eds.): Deutsche Sprache in der Slowakei II. Geschichte, Gegenwart und Didaktik. Wien: 203-218.

Šajánková, Monika (2005a): Gebrauch, Erwerb und Verständnis von Phraseologismen. Dissertation an der Philosophischen Fakultät der Comenius Universität Bratislava.

Šajánková, Monika (2005b): "Auswahl der Phraseologismen zur Entwicklung der aktiven phraseologischen Kompetenz". In: Jankovičová, Milada/Mlacek, Jozef/Skladaná, Jana (eds.): Frazeologicke študie IV. Bratislava, Veda, Vydatel'stvo Slovenskej Akademie Vied: 325-340.

Šajánková, Monika (im Druck): "Phaseodidaktische Überlegungen zur Entwicklung der aktiven phraseologischen Kompetenz". In: Kržišnik, Erika (ed.): Phraseologie in der Sprachwissenschaft und anderen Disziplinen. Tagungsband EUROPHRAS Slovenija 2005.

Schindler, Franz (1998): "Ergebnisse einer empirischen Untersuchung zur Kenntnis tschechischer Sprichwörter (im Vergleich zur Simrock-Untersuchung von Chlosta et al.)". 
In: Hartmann, Dietrich (ed.): "Das geht auf keine Kuhhaut". Arbeitsfelder der Phraseologie. Bochum: 279-310. (= Studien zur Phraseologie und Parömiologie 16).

Steyer, Kathrin (2003): "Korpus, Statistik, Kookkurrenz. Lässt sich Idiomatisches "berechnen"?" In: Burger, Harald/Häcki Buhofer, Annelies/Gréciano, Gertrud (eds.): Flut von Texten - Vielfalt der Kulturen. Baltmannsweiler: 33-46. (= Phraseologie und Parömiologie 14).

Wotjak, Barbara (2001): "Phraseologismen im neuen Lernerwörterbuch - Aspekte der Phraseologiedarstellung im de Gruyter-Wörterbuch Deutsch als Fremdsprache". In: Häcki Buhofer, Annelies/Burger, Harald/Laurent, Gautier (eds.): Phraseologiae Amor. Aspekte europäischer Phraseologie. Baltmannsweiler: 263-279. (= Phraseologie und Parömiologie 8).

Wotjak, Barbara/Richter, Manfred (1993): Sage und schreibe. Deutsche Phraseologismen in Theorie und Praxis. Leipzig etc. 

für Deutsch als Fremdsprache.

\section{Anhang: 143 Phraseologismen im Kernbereich des phraseologischen Optimums}

\begin{tabular}{|c|c|c|c|}
\hline Komponente & Phraseologismus & $\begin{array}{l}\text { bekannt - } \\
\text { verwendet }\end{array}$ & Frequenz \\
\hline Abfuhr & jm eine Abfuhr erteilen & 84 & 574 \\
\hline Ahnung & keine (blasse) Ahnung (von etw.) haben & 82 & 2333 \\
\hline Anhieb & auf Anhieb & 100 & 2211 \\
\hline Arm & jm (mit etw.) unter die Arme greifen & 90 & 1054 \\
\hline Atem & jn/etw. in Atem halten & 77 & 970 \\
\hline Auge & jm in die Augen/ins Auge springen/fallen & 81 & 1733 \\
\hline Auge & jn/etw. im Auge behalten/haben & 98 & 4062 \\
\hline Auge & jn/etw. (nicht) aus den Augen/aus dem Auge verlieren & 96 & 4595 \\
\hline Auge & etw. ins Auge fassen & 80 & 1710 \\
\hline Ball & am Ball bleiben/sein & 85 & 924 \\
\hline Bein & auf den Beinen sein & 89 & 759 \\
\hline Bein & etw. auf die Beine stellen & 87 & 912 \\
\hline Bescheid & Bescheid wissen (über jn/etw.) & 97 & 1907 \\
\hline Bild & $\operatorname{sich}_{\text {Dativ }}$ ein Bild von jm/etw. machen & 87 & 537 \\
\hline Bildfläche & von der Bildfläche verschwinden & 89 & 565 \\
\hline Bühne & etw. über die Bühne bringen & 81 & 3933 \\
\hline Dach & unter Dach und Fach sein & 92 & 1711 \\
\hline Dach & etw. unter Dach und Fach bringen & 78 & 1711 \\
\hline Dorn & jm ein Dorn im Auge sein & 82 & 1347 \\
\hline Druck & jn unter Druck setzen & 79 & 9741 \\
\hline Dunkel & im Dunkeln tappen & 85 & 751 \\
\hline Ecke & jn um die Ecke bringen & 91 & 2129 \\
\hline Eis & etw. auf Eis legen & 96 & 1999 \\
\hline Faust & etw. auf eigene Faust tun & 99 & 1202 \\
\hline Ferse & jm (dicht) auf den Fersen bleiben/sein & 93 & 554 \\
\hline Finger & jm auf die Finger sehen/schauen/gucken & 93 & 633 \\
\hline Fußstapfen & in js Fußstapfen treten & 83 & 706 \\
\hline gang & gang und gäbe sein & 90 & 623 \\
\hline Gewicht & ins Gewicht fallen & 78 & 1639 \\
\hline Griff & etw. in den Griff bekommen/kriegen & 91 & 3399 \\
\hline Griff & jn/etw. im Griff haben & 88 & 3032 \\
\hline Großen & im Großen und Ganzen & 99 & 1021 \\
\hline Grund & etw. auf den Grund gehen & 93 & 812 \\
\hline
\end{tabular}




\begin{tabular}{|c|c|c|c|}
\hline Haar & $\operatorname{sich}_{\text {Akk. }}$ in die Haare geraten/kriegen & 87 & 517 \\
\hline Hals & jm um den Hals fallen & 94 & 1588 \\
\hline Hand & alle Hände voll zu tun haben & 95 & 651 \\
\hline Hand & etw. (selbst) in die Hand nehmen & 95 & 1922 \\
\hline Hand & (klar) auf der Hand liegen & 93 & 2696 \\
\hline Hand & jm (völlig) freie Hand lassen & 88 & 863 \\
\hline Hand & js rechte Hand sein & 83 & 1031 \\
\hline Hand & Hand in Hand (arbeiten) & 80 & 1162 \\
\hline Hand & $\operatorname{sich}_{\text {Dativ }}$ die Hände reiben & 79 & 603 \\
\hline Hand & etw. (nicht) aus der Hand geben & 78 & 2560 \\
\hline Hand & jm an die/zur Hand gehen & 77 & 1214 \\
\hline Hand & jm sind (die) Hände (und Füße) gebunden/gefesselt & 76 & 675 \\
\hline Handtuch & das Handtuch werfen/schmeißen & 77 & 517 \\
\hline Handwerk & jm das Handwerk legen & 76 & 507 \\
\hline Haufen & etw. über den Haufen werfen & 79 & 750 \\
\hline Häuschen & (ganz/völlig) aus dem Häuschen sein/geraten & 90 & 615 \\
\hline Herz & jm am Herzen liegen & 96 & 1822 \\
\hline Herz & von ganzem Herzen & 95 & 656 \\
\hline Herz & jn in sein/ins Herz schließen & 89 & 1163 \\
\hline Himmel & aus heiterem Himmel & 95 & 606 \\
\hline Hin & nach vielem/langem/einigem/ewigem Hin und Her & 94 & 4583 \\
\hline Höhe & $\begin{array}{l}\text { (nicht) (ganz) auf der Höhe sein; sich auf der Höhe } \\
\text { fühlen }\end{array}$ & 83 & 1491 \\
\hline Hose & in die Hose gehen & 84 & 622 \\
\hline Hose & sich (vor Angst) in die Hosen machen & 79 & 622 \\
\hline Hut & jn/etw./alle(s) unter einen Hut bringen & 83 & 861 \\
\hline Kauf & etw./jn (mit) in Kauf nehmen & 91 & 5319 \\
\hline Keim & etw. im Keim ersticken & 76 & 609 \\
\hline Kippe & auf der Kippe stehen/sein & 94 & 848 \\
\hline Klaren & sich $_{\text {Dativ }}$ über etw. im Klaren sein & 96 & 1417 \\
\hline Kopf & sich etw. durch den Kopf gehen lassen & 97 & 896 \\
\hline Kopf & $\operatorname{sich}_{\text {Dativ }}$ etw. in den Kopf setzen & 91 & 1261 \\
\hline Kopf & jm etw. an den Kopf werfen & 83 & 838 \\
\hline Kopf & jn vor den Kopf stoßen & 81 & 703 \\
\hline Kosten & auf seine Kosten kommen & 90 & 1908 \\
\hline Kragen & jm an den Kragen gehen & 82 & 707 \\
\hline Kürzeren & den Kürzeren ziehen & 90 & 805 \\
\hline Länge & $\operatorname{sich}_{\text {Akk. }}$ in die Länge ziehen & 89 & 627 \\
\hline
\end{tabular}



für Deutsch als Fremdsprache.

\begin{tabular}{|c|c|c|c|}
\hline Last & jm zur Last fallen & 89 & 2400 \\
\hline Laufenden & (mit etw.) auf dem Laufenden sein & 92 & 616 \\
\hline Leben & ums Leben kommen & 90 & 10971 \\
\hline Licht & grünes Licht geben/erhalten & 79 & 3330 \\
\hline Licht & etw. ans Licht bringen & 77 & 1584 \\
\hline Lorbeer & $\left(\operatorname{sich}_{\mathrm{Akk}}\right)$ auf den/seinen Lorbeeren ausruhen & 86 & 501 \\
\hline Luft & in der Luft liegen & 88 & 4690 \\
\hline Luft & (gleich/schnell/leicht) in die Luft gehen & 83 & 4460 \\
\hline Luft & aus der Luft gegriffen sein & 82 & 1965 \\
\hline Luft & in der Luft schweben/hängen & 76 & 4690 \\
\hline Mann & etw. an den Mann bringen & 78 & 851 \\
\hline Mann & (s)einen Mann stehen & 76 & 2447 \\
\hline Mond & hinter/auf dem Mond leben & 91 & 677 \\
\hline Mund & jm etw. in den Mund legen & 80 & 1853 \\
\hline Naht & aus den/allen Nähten platzen/gehen & 89 & 702 \\
\hline Nase & (von jm/etw.) die Nase (gestrichen) voll haben & 93 & 813 \\
\hline Nase & auf die Nase fallen (mit etw.) & 84 & 534 \\
\hline Nase & jn an der Nase herumführen & 81 & 569 \\
\hline Nenner & etw. auf einen (gemeinsamen) Nenner bringen & 76 & 524 \\
\hline Nerv & jm auf die Nerven fallen/gehen & 97 & 943 \\
\hline Partie & mit von der Partie sein & 88 & 1702 \\
\hline Pech & Pech haben & 98 & 707 \\
\hline Punkt & etw. auf den Punkt bringen & 94 & 2710 \\
\hline $\operatorname{Rad}$ & unter die Räder kommen/geraten & 81 & 794 \\
\hline Rahmen & aus dem Rahmen fallen & 87 & 623 \\
\hline Rede & jn zur Rede stellen & 91 & 709 \\
\hline Rede & jm Rede und Antwort stehen & 88 & 1244 \\
\hline Reihe & an der Reihe sein/an die Reihe kommen & 96 & 1724 \\
\hline Reihe & aus der Reihe tanzen & 95 & 669 \\
\hline Rolle & eine/keine (große/kleine) Rolle spielen & 95 & 5224 \\
\hline Rücken & jm in den Rücken fallen & 89 & 1049 \\
\hline Rücken & jm/etw. den Rücken kehren/wenden/zuwenden/zudrehen & 84 & 1810 \\
\hline Runde & über die Runden kommen & 91 & 1183 \\
\hline Schach & jn in Schach halten & 80 & 868 \\
\hline Schatten & jn/etw. in den Schatten stellen & 77 & 1244 \\
\hline Schlange & Schlange stehen & 93 & 1277 \\
\hline Schliche & jm auf die Schliche kommen & 84 & 556 \\
\hline
\end{tabular}




\begin{tabular}{|c|c|c|c|}
\hline Schuhe & jm (die Schuld an) etw. in die Schuhe schieben & 85 & 549 \\
\hline schwarz & (etw.) schwarz auf weiß (haben/besitzen) & 94 & 573 \\
\hline Schwarze & ins Schwarze treffen & 88 & 528 \\
\hline Schwung & in Schwung kommen & 90 & 1579 \\
\hline Spiel & auf dem Spiel stehen & 89 & 2341 \\
\hline Spiel & etw. aufs Spiel setzen & 89 & 1599 \\
\hline Spitze & etw. auf die Spitze treiben & 85 & 687 \\
\hline Sprache & etw. zur Sprache bringen & 86 & 2177 \\
\hline Spur & jm/etw. auf die Spur kommen & 79 & 2497 \\
\hline Staub & $\operatorname{sich}_{\text {Akk. }}$ aus dem Staub(e) machen & 90 & 518 \\
\hline Stelle & auf der Stelle treten/nicht von der Stelle kommen & 83 & 1742 \\
\hline Stern & (noch) in den Sternen stehen & 91 & 581 \\
\hline Stich & jn (nicht) im Stich lassen & 94 & 1938 \\
\hline Strang & an einem/am gleichen/selben Strang ziehen & 82 & 979 \\
\hline Straße & auf der Straße sitzen/stehen/liegen & 81 & 10185 \\
\hline Straße & jn auf die Straße setzen/werfen & 80 & 5933 \\
\hline Strich & $\begin{array}{l}\text { jm/etw. einen (dicken) Strich durch die Rechnung } \\
\text { machen }\end{array}$ & 90 & 637 \\
\hline Tag & Tag und Nacht & 97 & 1788 \\
\hline Tasche & etw. (schon) in der Tasche haben & 79 & 2610 \\
\hline Teufel & zum Teufel gehen/sein & 76 & 563 \\
\hline Tisch & unter den Tisch fallen & 82 & 837 \\
\hline Tisch & jn über den Tisch ziehen & 81 & 1004 \\
\hline Ton & den Ton angeben & 85 & 608 \\
\hline Tour & auf Touren kommen & 84 & 879 \\
\hline Trab & jn auf Trab bringen & 82 & 790 \\
\hline Tür & jn vor die Tür setzen & 86 & 1268 \\
\hline Wand & in seinen vier Wänden & 90 & 893 \\
\hline Wasser & jn/sich über Wasser halten & 88 & 1044 \\
\hline Weg & jm über den Weg laufen & 93 & 943 \\
\hline Weite & das Weite suchen & 82 & 814 \\
\hline Wort & (immer) das letzte Wort haben/behalten (wollen/müssen) & 95 & 2114 \\
\hline Wort & jn beim Wort nehmen & 90 & 595 \\
\hline Zahl & rote Zahlen schreiben; in den roten Zahlen sein & 80 & 2670 \\
\hline Zeile & zwischen den Zeilen lesen (können) & 90 & 581 \\
\hline Zeug & $\operatorname{sich}_{\text {Akk. }}$ (tüchtig) ins Zeug legen & 80 & 711 \\
\hline
\end{tabular}

